\title{
Factores externos en el desempeño de pruebas genéricas de Estado (SaberPro) de inglés en estudiantes de administración y afines en Colombia
}

\author{
Leonor Cabeza, Jahir Lombana y Jaime Castrillón
}

\section{RESUMEN}

Existe una política para fortalecer el bilingüismo en Colombia, cuyos resultados en las pruebas estatales no son los mejores. Se requiere investigar los factores que puedan influir en esa dinámica. Esta investigación busca explicar y clasificar los resultados de las pruebas genéricas de Estado en las instituciones de educación superior (SaberPro), en el módulo de inglés, en los programas de administración y afines en Colombia. Se utilizó un conjunto de variables independientes extrínsecas (económicas, familiares e institucionales), a las cuales se llegó mediante un proceso iterativo. El método usado fue el de regresión logística binaria, donde el éxito radica en obtener una puntuación menor o igual a la media en el módulo de inglés. Las variables seleccionadas tienen relación con el resultado de las pruebas de inglés, pero se recomiendan estudios adicionales involucrando variables intrínsecas.

Palabras clave: administración de empresas, bilingüismo, pruebas nacionales, Colombia.

Leonor Cabeza

Colombiana. Maestra en Administración de Empresas, Universidad del Norte, Barranquilla, Colombia. Profesora Asistente, Universidad del Norte, Barranquilla, Colombia. Temas de investigación: competencias gerenciales, desarrollo curricular, estadística y matemáticas para administración, modelación para toma de decisiones.

Jahir Lombana

lombanaj@uninorte.edu.co

Colombiano. Doctor en Economía, Universidad del Goettingen, Alemania. Profesor Asociado, Universidad del Norte, Barranquilla, Colombia. Temas de investigación: competencias gerenciales, acreditaciones en instituciones de educación superior, desarrollo curricular, competitividad. 
Fatores externos no desempenho de provas genéricas do Estado (SaberPro) de inglês em estudantes de administração e afins na Colômbia

\title{
RESUMO
}

Existe uma política para fortalecer o bilinguismo na Colômbia, cujos resultados nas provas estatais não são os melhores. É necessário pesquisar os fatores que possam influir nessa dinâmica. Esta pesquisa procura explicar e classificar os resultados das provas genéricas do Estado nas instituições de educação superior (Saber Pro), no módulo de inglês, nos programas de administração e afins na Colômbia. Se utilizou um conjunto de variáveis independentes extrínsecas (econômicas, familiares e institucionais), às quais se obteve por meio de um processo iterativo. O método usado foi o de regressão logística binaria, onde o êxito radica em obter uma pontuação menor ou igual à media no módulo de inglês. As variáveis selecionadas têm relação com o resultado das provas de inglês, más é recomendável estudos adicionais envolvendo variáveis intrínsecas.

Palavras chave: administração de empresas, bilinguismo, provas nacionais, Colômbia.

\section{External factors in the performance of generic State tests (SaberPro) of English in} administration and related students in Colombia

\begin{abstract}
There is a policy to strengthen bilingualism in Colombia, whose results in the state tests are not the best. So it is necessary to investigate the factors that may influence this dynamic. This research seeks to explain and classify the results of the generic state tests in higher education institutions (Saber Pro), in the English module, in administration and related programs in Colombia. A set of extrinsic independent variables (economic, family and institutional) were used, drawn up through an iterative process. The method used was binary logistic regression, where the success lies in obtaining a score less than or equal to the average in the English module. The selected variables are related to the English test results, but additional studies involving intrinsic variables are recommended.
\end{abstract}

Key words: business administration, bilingualism, national tests, Colombia. 


\section{Introducción}

El aprendizaje de una segunda lengua se ha convertido en prioridad en la educación colombiana (Ministerio de Educación Nacional (MEN), 2014; Cárdenas y Miranda, 2014; Fandiño, Bermúdez y Lugo, 2012), lo anterior, como estrategia adicional a las cuatro prioridades planteadas en el plan de desarrollo 2014-2018 (República de Colombia, 2014) para mejorar el sistema educativo: Jornada Única, Excelencia Docente, Colombia Libre de Analfabetismo, Calidad en la Educación Superior y la que aquí se analiza, Colombia Bilingüe. Esta estrategia busca pasar de $2 \%$ a 8\% en el nivel B1 (Preintermedio) y del 7\% al 35\% en el nivel A2 (Básico) (MEN, 2014). Una de las mediciones de la evolución en esta política se puede dar en las pruebas llamadas Saber, que se realizan en diferentes niveles educativos. Este trabajo se centra en las pruebas en educación superior, llamadas SaberPro y particularmente en la prueba genérica de inglés para los estudiantes en administración y afines. El objetivo de la investigación es explicar y clasificar los resultados de las pruebas del SaberPro de los programas de administración y afines en el módulo de inglés, a partir de un conjunto de variables independientes. Después de esta introducción, en la que se hace una revisión de la literatura referente a los factores que se han tenido en cuenta para el aprendizaje de una segunda lengua, y particularmente el estudio del inglés en las disciplinas administrativas, en la segunda parte se plantea la metodología como una investigación multivariable, la cual utiliza la regresión logística que permite modelar la probabilidad de un evento ocurrido como función de otros factores usando el método de máxima verosimilitud, el que asume independencia entre las observaciones y la ausencia de error en la medida de las variables. En la tercera parte se presenta la discusión y resultados de acuerdo con el análisis del modelo, con los factores y sus probabilidades de ocurrencia. Finalmente, el artículo presenta las conclusiones.
El aprendizaje de una segunda lengua parte de la voluntad misma de quien la quiere aprender. El trabajo sistemático de Gardner (1983, 1985), dio pie para estudiarlo desde una necesidad meramente psicológica; sin embargo, la literatura ha profundizado otros factores, tanto internos, como externos, que pueden hacer que el aprendizaje se dé de manera más o menos fluida. De los trabajos más citados en cuanto a los factores que influencian el aprendizaje de una lengua, están los de Mitchell y Myles (2004) y Lightbown y Spada (2006), los cuales mencionan: edad, personalidad, motivación (intrínseca), experiencias, capacidad cognitiva y aprendizaje de lenguas similares (por ejemplo, entre holandés e inglés). Todos estos factores clasificados como internos. Sin embargo, factores externos como formas de enseñar el idioma (currículo e instrucción), cultura y estatus, motivación (extrínseca) y acceso a hablantes nativos, pueden también influir en el aprendizaje (Della Chiesa, Scott y Hinton, 2012). Precisamente en los factores externos que puedan influenciar el desempeño del aprendizaje del inglés, se inscribe este trabajo.

$\mathrm{Al}$ centrarse sólo en las motivaciones de aprender una lengua diferente a la nativa y tomando únicamente la motivación extrínseca, el trabajo de Chiswick (2008), con respecto al desempeño en la lengua extranjera de inmigrantes, permitió clasificar factores tales como: exposición a la lengua extranjera, eficiencia en el aprendizaje e incentivos económicos. En este último factor, los beneficios de la inversión deben compensar los costos. Siguiendo a Rodríguez-Chamussy, LopezCalva y Miyamoto (2012), el análisis de costo-beneficio en el aprendizaje de una segunda lengua debe tratarse de manera equivalente al capital humano, que es: 1) intangible y no transferible, 2) al ser creado se sacrifican recursos (en tiempo y financieros) y 3) los retornos se ven en el mercado laboral. Esta perspectiva, aunque más por el efecto de aprender una lengua, sirve para justificar la importancia de revisar cómo se apoya la política pública en los factores que mejoran el desempeño de estudiantes en una segunda lengua. 
Así, los trabajos de Tainer (1988) y McManus, Gould y Welch (1983), confirman que el dominio de una segunda lengua mejora los salarios en inmigrantes. En general, la investigación en aspectos socioeconómicos como determinantes en el aprendizaje de una segunda lengua han sido poco estudiados (Lamb 2012; Kormos y Kiddle 2013), pero hay resultados como los de Nikolov (2009) y PISA (2013), que encuentran en los antecedentes académicos de los padres, correlaciones con los resultados de sus hijos.

Un trabajo de Valenzuela, Romero, Vidal-Silva y Philominraj (2016), permite reconocer que a pesar de las nuevas herramientas tecnológicas de enseñanza de idiomas y la procedencia de los estudiantes, el profesor sigue siendo importante en los resultados de desempeño de una segunda lengua. El estudio realizado para estudiantes en Chile, muestra que la mediación de las tecnologías de la información y la comunicación (TIC) debe tener un acompañamiento para lograr los objetivos de aprendizaje, sin embargo, esto dependerá también del tipo de instrucción que dé el docente (Richards y Rodgers 2001; RuedaCataño y Wilburn, 2014; García-Esteban, 2013). En otro estudio de naturaleza cuantitativa - los cuales siguen siendo escasos - realizado para estudiantes en México (Santana, García-Santillán y EscaleraChávez, 2016), aparece el tiempo de dedicación, la institución (tipo de escuela) y frecuencia de lectura, como variables relevantes que afectan el desempeño, y también variable de percepción de habilidades: creer que se habla, se lee, se escribe, se entiende bien. Así también, el venir de instituciones privadas mejora ostensiblemente los resultados, esta conclusión la comparten para Colombia MejíaMejía (2016) y Sánchez-Jabba (2013) en educación secundaria. En estos estudios, variables como ingreso familiar, jornada educativa (mañana, tarde, noche) y acceso a internet son consideradas, aproximándose a los resultados que se presentan más adelante en este documento para educación superior.

Un aspecto fundamental en el análisis de los factores que afectan el aprendizaje de una segunda lengua, es la razón de aprender. El concepto de lenguaje para propósitos específicos (del inglés Language for Specific Purposes-LSP), resulta particularmente importante en las áreas administrativas, donde el aprendizaje de un segundo idioma se ha convertido en competencia intercultural ineludible (Zhou y Griffiths, 2011 y Nteliou y Kehagia, 2016), particularmente en lo que respecta al idioma inglés en un contexto de linqua franca ${ }^{1}$ (Nickerson, 2005; Charles, 2007; Rogerson-Revell, 2007; Kankaanranta y Louhiala-Salminen, 2013). En los noventa el estudio del inglés como linqua franca apenas si se hacía notar (St. John, 1996), pero en el nuevo mileno, el interés por su estudio se ha materializado, haciéndose necesario ahondar en la investigación respecto a la necesidad del idioma en disciplinas particulares (inglés para negocios, inglés para medicina, etcétera) y revisar la relación existente entre los resultados de pruebas y factores que influencian en estos casos. Es necesario, por ejemplo, poner énfasis en la coherencia entre lo que aprende un estudiante y lo que requiere la industria (Waner, 1995; Bacha y Bahous, 2008; Zhang, 2013), que muchas veces no es lo que se evalúa en pruebas genéricas. Asimismo, profundizar sobre las necesidades por aprender un idioma nuevo para un estudiante de negocios frente al de otra disciplina, que según algunos estudios (Trinder, 2013; Trinder y Herles, 2011; Moody, 1988) puede facilitarse debido a la característica extrovertida de quienes la estudian. Adicionalmente la evolución de las relaciones económicas entre países van de la mano con las necesidades idiomáticas de los futuros egresados que deben incluso redefinir la forma como se enseña una lengua (Fuertes-Olivera y Gómez-Martínez, 2004; Bhatia y Bremner, 2012; Zagan-Zelter y Zagan-Zelter, 2010; Zhang, 2013; Kankaanranta y

\footnotetext{
${ }^{1}$ Knapp y Meierkord (2002) consideran su origen en los siglos XV y XIX en la Costa Sur-Oriental del Mediterráneo, como una lengua que, sin ser lengua nativa, a través de un arreglo de lenguaje les permitía comunicarse para comerciar.
} 
Louhiala-Salminen, 2013) y cómo ésta se evalúa para distintos contextos.

\section{Metodología}

Los resultados de estas pruebas fueron clasificados en dos grupos: los estudiantes que obtuvieron puntuaciones superiores al promedio y los que obtuvieron puntuaciones menores e iguales al promedio; variable dicotómica $\mathrm{Y}=0.1$ respectivamente. Para este trabajo se considera como éxito el tener una puntuación en inglés, menor o igual a la media, que es el problema que se quiere explicar y clasificar. La regresión logística binaria permite modelar la probabilidad de un evento ocurrido como función de otros factores usando el método de máxima verosimilitud que asume independencia entre las observaciones y la ausencia de error en la medida de las variables. El modelo de regresión logística se puede expresar por (Reyes, Escobar, Duarte y Ramírez, 2007):

$$
\begin{gathered}
\log [\pi /(1-\pi)]=\operatorname{logit}(\pi)= \\
\beta_{0}+\beta_{1} X_{1}+\beta_{2} X_{2}+\beta_{3} X_{3}+\cdots \ldots \ldots+\beta_{k} X_{k}
\end{gathered}
$$

Donde $\pi$ es la probabilidad de que ocurra el evento de interés; obtener una puntuación en el módulo inferior o igual a la media o puntuación en el módulo superior a la media, dependiendo de los valores de las variables independientes $X_{i}$. Los $\beta_{i}$ representa la diferencia en el cambio del logit por unidad de $X_{i}$. El valor logit para $X_{i}=0$, es la constante $\beta_{0} y$ el valor logit para puntuación categórica $X_{i}=1$, es $\beta_{0}$ más los coeficientes $\beta_{\mathrm{i}}$.

Los coeficientes de la variable dependiente se pueden transformar en probabilidades, la función exponencial:

$$
\left.\operatorname{odds}(\pi)=\mathrm{e}_{0}^{\beta}+\beta_{1} \mathrm{X}_{1}+\beta_{2} \mathrm{X}_{2}+\beta_{3} \mathrm{X}_{3}+\cdots \ldots \ldots+\beta \mathrm{kXk}\right)
$$

Una variable categórica puede tomar el valor $0 \mathrm{o}$ 1 ; no tener la condición es $\mathrm{X}_{\mathrm{i}}=0$ y toma como valor la constante. odds $(\pi)$ de las categorías de referencia $X_{i}=0$; con puntuación menor o igual a la media $=\mathrm{e}^{\beta}{ }_{0}$ Si Xi=1:

odds $(\pi)$ de $\mathrm{X}_{\mathrm{i}}=1$,

con puntuación menor o igual a la media $=$

$$
\left.\mathrm{e}^{\beta}{ }_{0}+\beta_{1} \mathrm{X}_{1}+\beta_{2} \mathrm{X}_{2}+\beta_{3} \mathrm{X}_{3}+\cdots \ldots . .+\beta_{\mathrm{k}} \mathrm{X}_{\mathrm{k}}\right)
$$

Una razón de probabilidades (en adelante OR por el inglés odd ratio) es una medida del tamaño del efecto de un evento. Esto es, la proporción de la probabilidad de que ocurra un evento en un grupo entre las probabilidades de que ocurra en otro grupo. Lo cual es importante para interpretar los resultados, especialmente para la variable categórica. El modelo también se puede expresar mediante la función logística (Berlanga-Silvente y Vilà-Baños, 2014):

$$
\begin{aligned}
\mathrm{P}(\mathrm{Y}=\mathrm{i}) \pi= & 1 /(1+\mathrm{e}-(\beta 0+\beta 1 \mathrm{X} 1+\beta 2 \mathrm{X} 2+\beta 3 \mathrm{X} 3+\ldots \\
& +\beta \mathrm{kXk}) ; \text { donde } \mathrm{i}=0,1 \quad(3)
\end{aligned}
$$

Calcula la probabilidad que un individuo que presente ciertos valores de $\mathrm{X}_{\mathrm{i}}$ tenga una puntuación igual o inferior a la media, en caso contrario es la probabilidad de tener puntuación superior a la media bajo iguales condiciones. Conociendo los valores de las variables independientes se puede calcular la probabilidad de que ocurra el evento de interés si tienen esos valores de $\mathrm{X}_{\mathrm{i}}$. Para los OR de cada variable menores a 1 , los $\beta$ son negativos, luego hay una relación inversa en el logit $(\pi)$ y representa un porcentaje decreciente del OR; si $\beta>1$ se presenta un relación directa con el $\operatorname{logit}(\pi)$ y representa un porcentaje de incremento en los OR, comparado con el de la categoría de referencia.

Los requisitos y etapas a seguir son (Berlanga-Silvente y Vilà-Baños, 2014):

- Recodificar las variables independientes categóricas u ordinales en variables ficticias dummy, con valores 0 o 1, al igual que la variable dependiente.

- Evaluar el efecto de confusión y de interacción de los modelos 
- Evaluar la bondad de ajuste de los modelos.

- Analizar la fuerza, sentido y significación de los coeficientes $(\beta)$ y sus OR $\left(e^{\beta}\right)$; y estadístico de prueba.

El método de máxima verosimilitud de probabilidad, que es una probabilidad condicional y un proceso iterativo, hasta encontrar el mejor modelo ajustado a los datos. El SPSS utiliza la prueba de razón de verosimilitud tomando el doble del logaritmo natural de la probabilidad de Y (-2LL), estadístico que se comporta como una distribución Chi-cuadrado $\left(\mathrm{X}_{2}\right)$ :

$$
\begin{gathered}
\text { X2=-2LLN-(-2LLF) }= \\
\text {-2In (Probabilidad modelo nulo)/ } \\
\text { (Probabilidad modelo completo) }
\end{gathered}
$$

Si el p-valor $<0.05$ el modelo es significativo y predice a Y.

La prueba de la razón de verosimilitud es para el modelo, y el estadístico de Wald se usa para probar la significación de cada variable independiente, prueba la $\mathrm{H}_{0}=\beta_{\mathrm{i}}=0 ; \mathrm{H}_{1}=\beta_{\mathrm{i}} \neq 0$; el estadístico se comporta como una $\mathrm{X}^{2}$ (Chi cuadrado) y se espera que el p-valor $<0.05$, es decir que sea significativo, para no eliminar la variable. Para medir el porcentaje que explica el modelo, se utilizan dos medidas Cox y Snell $\mathrm{R}^{2}$, el estadístico debe ser menor a 1 para un modelo perfecto. Otro estadístico es Nagelkerke's $\mathrm{R}^{2}$ que debe tomar valores entre 0 y 1 . También se puede determinar el porcentaje de acierto del modelo para predecir, si el modelo es perfecto la tabla de clasificación presenta el $100 \%$ de aciertos en la diagonal. Si el modelo de regresión logística tiene homocedasticidad, el porcentaje de acierto puede ser igual en las filas.

Se partió de una población de 11085 resultados de exámenes de estudiantes, pero no todos diligenciaron el cuestionario de inscripción completo o correctamente; se trabajó con una muestra de 2148 datos y se validó el modelo en 938 casos. Para una población finita con un nivel de significancia del 5\% y margen de error del $1.9 \%$, se recomienda una muestra de 2 146, luego podemos aceptar que la muestra tomada representa el comportamiento de la población.

Las variables independientes pueden ser familiares y personales del estudiante que presentó el examen; información económica, del programa y de la institución educativa a los que pertenece. Esta información se recogió en el cuestionario que diligencian todos los estudiantes que deben presentar el examen para poder optar al título profesional. De esta encuesta se tomaron inicialmente 26 variables; para esto se verificó la independencia de las variables independientes mediante una Rho de Spearman, utilizando una prueba Chi cuadrado $\left(\mathrm{X}^{2}\right)$ a un nivel de significancia de 0.05 y la tolerancia y la VIF (Variance Inflation Factory), que es el inverso de la tolerancia y permite determinar la multicolínealidad. Se utilizó el SPSS 24 para el análisis estadístico de datos y el método de introducción.

\section{Análisis y resultados}

Antes de iniciar el proceso estadístico se agruparon algunas variables - una de ellas es el departamento de ubicación del programa- y se clasificaron en regiones geográficas: Andina, la cual comprende los departamentos de Antioquia, Boyacá, Caldas, Cundinamarca, Huila, Norte de Santander, Quindío, Risaralda, Santander y Tolima. Caribe, que incluye los departamentos de Atlántico, Bolívar, Cesar, Córdoba, Guajira, Magdalena y el archipiélago de San Andrés, Providencia y Santa Catalina. Región Pacífica, conformada por Choco, Valle del Cauca, Cauca y Nariño. Orinoquía, conformada por Meta, Vichada, Casanare y Arauca. Por último, la región Amazónica, que involucra Amazonas, Caquetá, Guainía, Guaviare, Putumayo y Vaupés.

Con el apoyo del SPSS 24 se realizó la codificación y definición de las variables dummy; es importante tener presente la categoría de referencia definida por cada variable. Si el SPSS no dispone de otra cosa, 
asume como referencia el último código; así se hizo en esta investigación excepto para género; tener beca; método del programa, carácter académico de la institución (ver tabla 1).

Para llegar al modelo se realizaron seis interacciones, lo cual involucra 10 variables independientes o predictoras, y se aplicó el estadístico Wald, el cual permitió ir eliminando variables del modelo hasta llegar al modelo que presentamos. A continuación se evalúa la bondad de ajuste de los modelos. Para evaluar la significancia global del modelo se utiliza el estadístico de la razón de Verosimilitud fórmula 4 (ver tabla 2).

Tabla 1. Variables categóricas de referencia

\begin{tabular}{|l|l|}
\hline \multicolumn{1}{|c|}{ Variables } & \multicolumn{1}{c|}{$\boldsymbol{X}_{\boldsymbol{i}}=\mathbf{0}$} \\
\hline Región Geográfica & Amazónica \\
\hline Educación del padre & Postgrado \\
\hline Ingresos familiares mensuales & 7 o más S.M.L.V \\
\hline Valor matricula-institución & Más de 5 millones de pesos \\
\hline Estudiante tienen beca & No \\
\hline Número de personas a cargo estudiante & 5 personas \\
\hline Estrato & 6 \\
\hline Genero del estudiante & Femenino \\
\hline Método del programa & Virtual \\
\hline Carácter académico de la institución & Institución tecnológica. \\
\hline
\end{tabular}

Fuente: construcción autores con SPSS 24.

Tabla 2. Significancia global del modelo

\begin{tabular}{|l|l|c|}
\hline$-2 \mathrm{LN}(\mathrm{LR})$ & Dato de SPSS - Bloque 0 & 2802.122 \\
\hline$-2 \mathrm{LN}(\mathrm{MV})$ & Dato de SPSS - Bloque 1 & 2207.977 \\
\hline Estadístico de Verosimilitud & $-2 \mathrm{LN}(\mathrm{R})-(-2 \mathrm{LN}(\mathrm{MV}))$ & 594.146 \\
\hline $\mathrm{gl}$ & Número de Variables Dummy tabla 5 & 38 \\
\hline $\mathrm{X}^{2}{ }_{38: 0.05}$ & Excel & 2148 \\
\hline $\mathrm{N}$ & Tamaño muestra & 53.38354062 \\
\hline $\mathrm{R}^{2}: \mathrm{R}$ cuadrado de Cox y Snell & & 0.242 \\
\hline Pseudo - $\mathrm{R}^{2}: \mathrm{R}$ cuadrado de Nagelkerke & $\mathrm{R}^{2} /\left(1-\mathrm{R}^{2}\right)$ & 0.332 \\
\hline
\end{tabular}

Fuente: construcción autores con SPSS 24. 
El estadístico de verosimilitud es superior al valor crítico de la Chi Cuadrado a un nivel de significancia del 0.05 (594.146>53.3854). Luego, concluimos que el valor de los $\beta_{\mathrm{i}}$ de las variables independientes del modelo es diferente a cero, y el modelo es significativo. Ambas pruebas estadísticas nos permiten afirmar que la probabilidad de que un estudiante obtenga una puntuación menor o igual a la media se relaciona con las variables: valor de la matrícula, el estudiante tiene beca, el número de personas a cargo, los ingresos familiares mensuales, el estrato, nivel de educación del padre, el género, la región donde se ubica el programa, el carácter académico de la institución y el método del programa.

Los valores de $\mathrm{R}^{2}=24.2 \%$ y Pseudo $-\mathrm{R}^{2}=33.2 \%$ aparentemente son bajos, y llevaría a pensar que la capacidad explicativa del modelo no es buena, pero de acuerdo con Morrison (1972), citado por Salas (1996), cuando las correlaciones se dan entre una variable dependiente binaria y se trata de modelos con variables cualitativas (dicotómicas o categóricas), que es el caso de esta investigación, para este tipo de variables es aceptable obtener estos resultados.

Existe otra prueba que permite medir la capacidad de clasificación del modelo encontrado, ésta es el área bajo la curva COR (ver gráfico 1) y la tabla de clasificación (ver tabla 3).

Este modelo presenta un 89.1\% (es decir, 1229/1379 100) de probabilidad de clasificar correctamente los puntajes menores o iguales a la media, es de decir, de sensibilidad y un $49.3 \%$ (es decir, 379/769 100) de probabilidad de clasificar correctamente los puntajes mayores a la media, es decir, de especificidad y clasifica correctamente el 74.9\% $((379+1229) / 2148 * 100)$ de los 2148 casos seleccionados y se valida en un muestra de 938 datos, donde muestra una sensibilidad del $87.3 \%$ y una especificidad del $46.7 \%$, con un $73 \%$ de datos del total, bien clasificados.

- Bajo el supuesto no paramétrico

- Hipótesis nula: área verdadera $=0.5$

$\mathrm{El}$ criterio que se asume para el área bajo la curva es, si $[0.5 ; 0.6]$ el modelo es malo; [ $0.6 ; 0.75]$ es regular; $[0.75 ; 0.90]$ es bueno; [0.90; 0.97] es muy bueno; $[0.97 ; 1]$ es excelente. En este caso el modelo presenta un área del $76 \%$ al $80 \%$ de intervalo de confianza con un 95\% de confianza, luego es bueno el modelo para clasificar (ver tabla 4).

Tabla 3. Clasificación

\begin{tabular}{|c|c|c|c|c|c|c|}
\hline & \multicolumn{6}{|c|}{ Pronosticado } \\
\hline & \multicolumn{3}{|c|}{ Casos seleccionados } & \multicolumn{3}{|c|}{ Casos no seleccionados } \\
\hline Observado & Mayor media & $\begin{array}{c}\text { Menor o igual } \\
\text { media }\end{array}$ & $\begin{array}{l}\text { Porcentaje } \\
\text { Correcto }\end{array}$ & Mayor media & $\begin{array}{c}\text { Menor o igual } \\
\text { media }\end{array}$ & $\begin{array}{c}\text { Porcentaje } \\
\text { Correcto }\end{array}$ \\
\hline Mayor media & 379 & 390 & 49.3 & 158 & 180 & 46.7 \\
\hline Menor o igual media & 150 & 1229 & 89.1 & 76 & 524 & 87.3 \\
\hline Porcentaje global & & & 74.9 & & & 72.7 \\
\hline
\end{tabular}

Fuente: construcción autores con SPSS 24. 
Tabla 4. Área bajo la curva

\begin{tabular}{|c|c|c|c|c|}
\hline \multicolumn{3}{|c|}{ Variables de resultado de prueba: } & \multicolumn{2}{c|}{ Probabilidad pronosticada } \\
\hline & & & \multicolumn{2}{|c|}{$\begin{array}{r}95 \% \text { de intervalo de confianza } \\
\text { asintótico }\end{array}$} \\
\cline { 4 - 5 } & & & Significación \\
\cline { 4 - 5 } & asintótica & Límite inferior & Límite superior \\
\hline 0.777 & 0.009 & 0.000 & 0.760 & 0.795 \\
\hline
\end{tabular}

Fuente: construcción autores con SPSS 24.

\section{Gráfica 1. Curva COR}

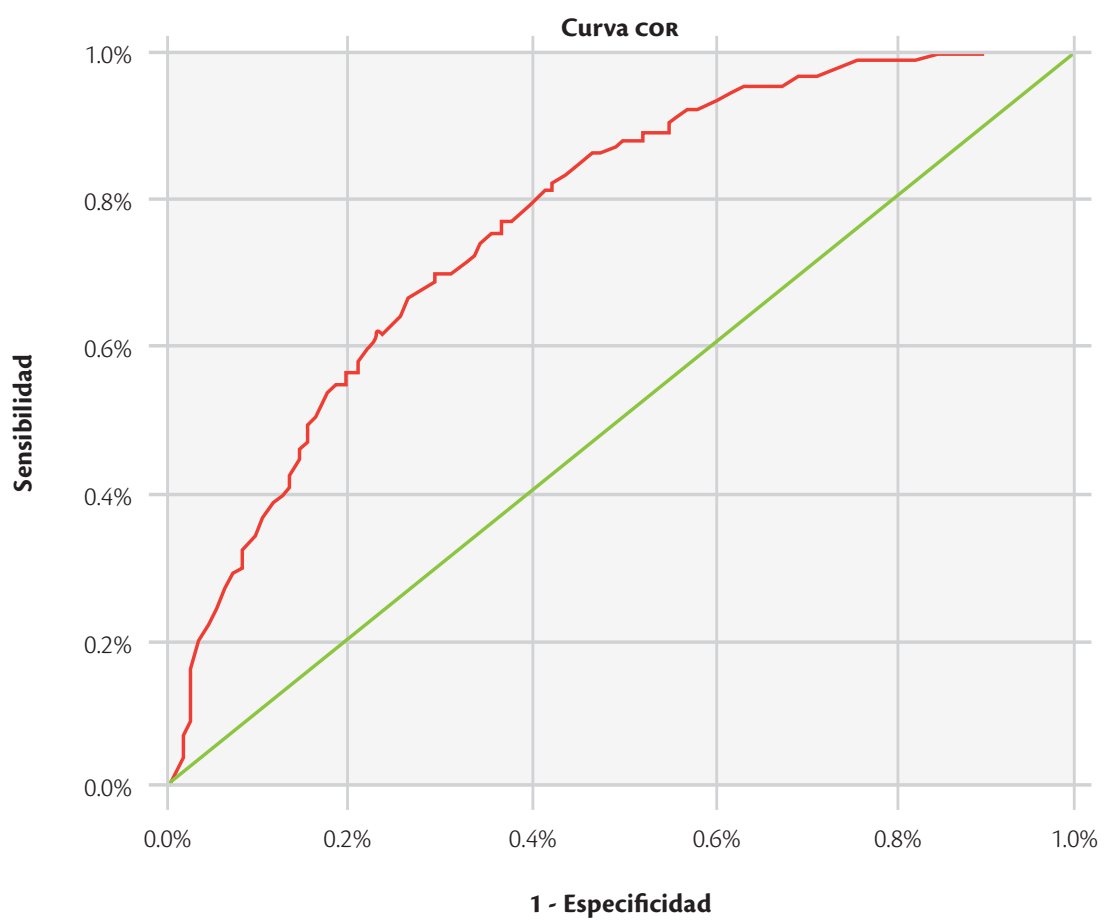

Los segmentos de diagonal se generan mediante empates 
Se determina el punto de corte (ver gráfica 1), es decir, donde se encuentra la pendiente de la curva, donde la especificidad y la sensibilidad toman sus máximos valores y encontramos el mayor porcentaje de puntajes menor e igual a la media y puntajes mayores a la media mejor clasificados; éste es 0.67086. Este valor se obtuvo de las coordenadas de la curva suministrada por el SPSS; corresponde a una sensibilidad del $69.1 \%$ y una especificidad del $71.8 \%$.

El modelo obtenido para el rendimiento en el módulo de Inglés, donde 1 es puntuación menor o igual a la media, y la puntuación 0 es superior a la media y los coeficientes $\beta_{\mathrm{i}}$ se toman de la tabla 5 ; mediante la fórmula 1 :

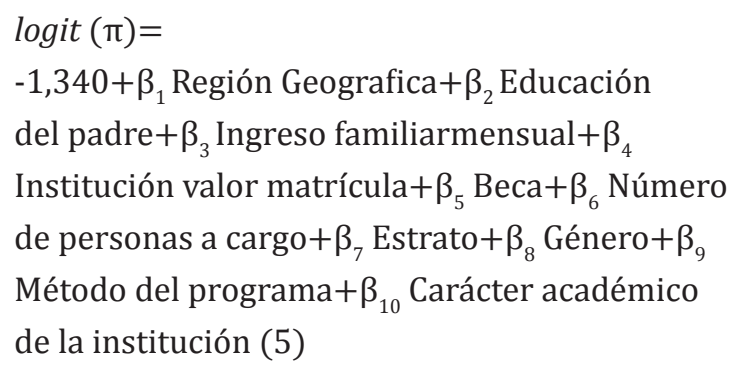

A continuación se analiza la fuerza, sentido y significación de los coeficientes $(\beta)$ y sus OR $\left(e^{\beta}\right)$, y estadístico de prueba. Observando los OR de cada variable independiente, hay un grupo de variables con valores mayores a 1 , lo que indica que hay una relación directa entre la variable dependiente y la independiente; ellas son: ingresos familiares; valor de la matrícula; carácter académico de la institución; estrato y educación del padre, que va desde no tener escolaridad a tener máximo educación técnica o tecnológica sin título. Para los OR con valores menores a 1, indica que hay una relación inversa, las variables son: estudiante con beca; número de personas a cargo; método del programa; género; regiones geográficas del programa; educación del padre con educación técnica o tecnológica con título, o profesional con o sin título y número de personas a cargo (tabla 5).
Con estos coeficientes y los OR, podemos calcular la probabilidad de tener en el módulo una puntuación menor o igual a la media; dependiendo de los valores de las $\mathrm{X}_{\mathrm{i}}$; si $\mathrm{X}_{\mathrm{i}}=0$, no tienen la característica, usando la formula 3 la probabilidad de tener puntuación menor o igual a la media para las variables categóricas de referencia se da por: $\pi=1 /\left(1+\mathrm{e}^{-} \beta_{0}\right)$ (tabla 6). Las probabilidades respectivas se calcularon utilizando la fórmula 3.

En la tabla 6 se observa la probabilidad de obtener una puntuación menor o igual a la media dependiendo de que $X_{i}=1$ o si $X_{i}=0$. Para valores de $X_{i}=0$, se calculó la probabilidad que el estudiante tenga una puntuación menor o igual a la media, esto es: si el estudiante es de la región Amazónica; educación del padre con posgrados; ingreso familiar mensual 7 SML o más; valor de la matrícula 5 millones de pesos o más; sin beca; con cinco o más personas a cargo; estrato 6; mujer; programa de carácter virtual e institución tecnológica; la probabilidad es de 0.208. Pero si mantenemos todas las características iguales y bajamos a cero el número de personas a cargo la probabilidad baja, es de 0.061. Luego, en este grupo de estudiantes es probable que tengan puntación superior a la media y entre menor número de personas a cargo, menor probabilidad de estar por debajo de la media.

Si el estudiante es de la región Pacífica; padre sin educación; ingresos familiares menor a 1 SMLV; paga matrícula entre 500 mil pesos y un millón de pesos; con beca; cero personas a cargo; estrato 1; hombre; programa presencial e institución universitaria, la probabilidad que tenga una puntuación en el módulo de inglés menor o igual a la media es de 0.838, modificando sólo la región geográfica por la región Andina o la Caribe se observa una probabilidad de 0.778 y 0.836 respectivamente, de que obtenga una puntuación menor o igual a la media, luego se concluye que los estudiantes de la región Andina tienen menor probabilidad que los del Caribe y Pacífica de tener rendimiento inferior a la media. 

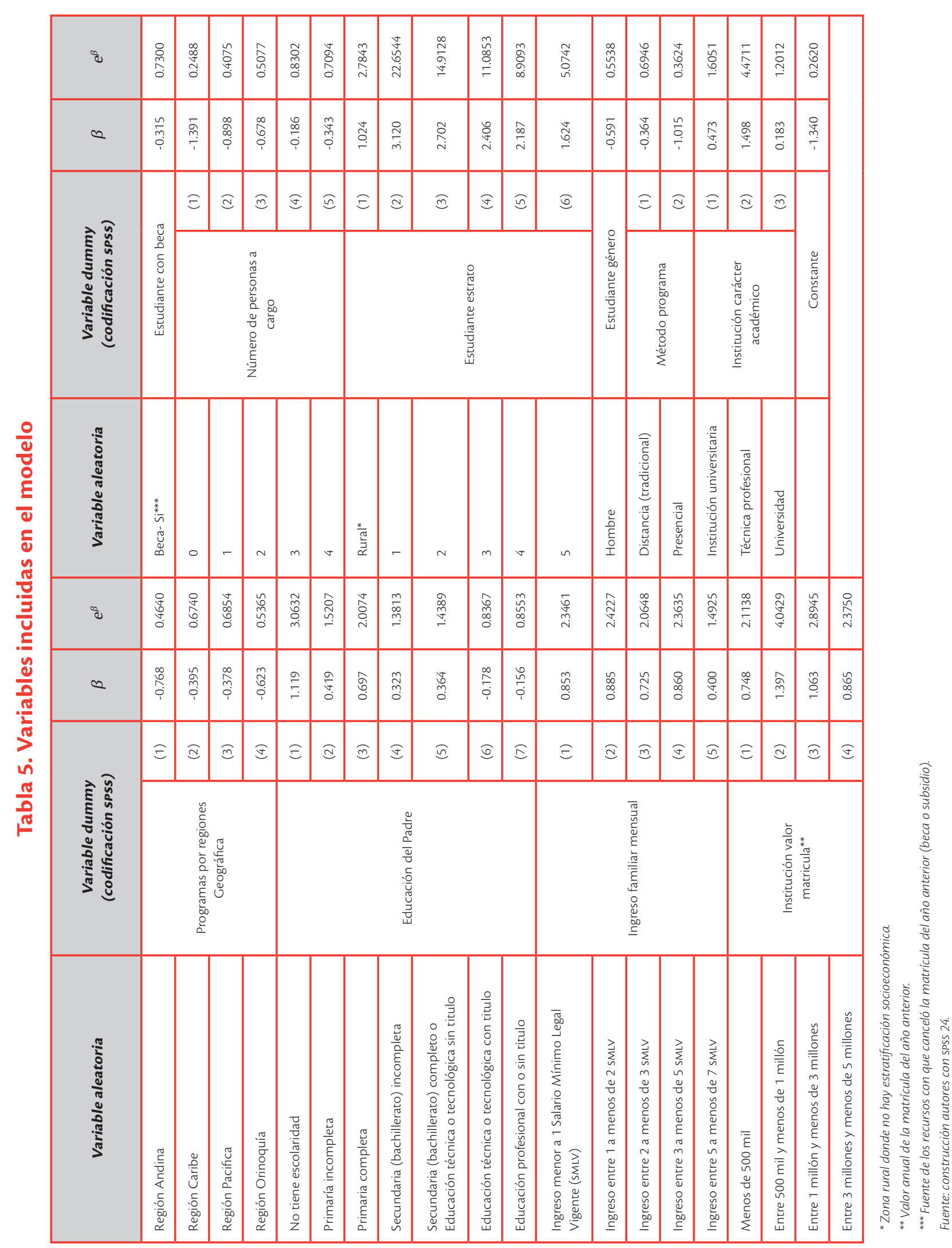
Tabla 6. Cálculo de probabilidades

\begin{tabular}{|c|c|c|c|c|c|c|c|c|c|c|c|c|c|c|}
\hline & 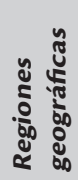 & 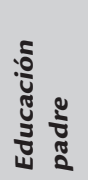 & 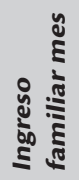 & $\frac{2}{\frac{\pi}{\pi}} \frac{\sqrt{\frac{\pi}{2}}}{\frac{\pi}{2}}$ & 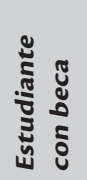 & 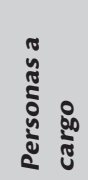 & 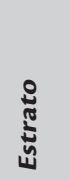 & 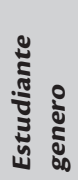 & 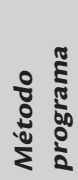 & 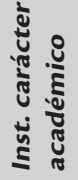 & 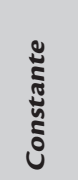 & $\begin{array}{l}\text { Е } \\
\frac{\pi}{3} \\
\text { हี } \\
\frac{1}{L}\end{array}$ & 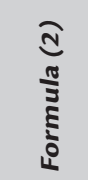 & 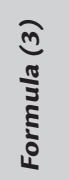 \\
\hline $\mathrm{Xi}$ & 0 & 0 & 0 & 0 & 0 & 0 & 0 & 0 & 0 & 0 & & & & \\
\hline$\beta X \mathrm{X}$ & & & & & & & & & & & -1.340 & -1.340 & 0.262 & 0.208 \\
\hline Xi & 0 & 0 & 0 & 0 & 0 & 1 & 0 & 0 & 0 & 0 & & & & \\
\hline$\beta X i$ & & & & & & -1.391 & & & & & -1.340 & -2.731 & 0.065 & 0.061 \\
\hline $\mathrm{Xi}$ & 3 & 1 & 1 & 2 & 1 & 1 & 2 & 1 & 2 & 3 & & & & \\
\hline$\beta X i$ & -0.378 & 1.119 & 0.853 & 1.397 & -0.315 & -1.391 & 3.120 & -0.591 & -1.015 & 0.183 & -1.340 & 1.644 & 5.174 & 0.838 \\
\hline $\mathrm{Xi}$ & 1 & 1 & 1 & 2 & 1 & 1 & 2 & 1 & 2 & 3 & & & & \\
\hline$\beta X i$ & -0.768 & 1.119 & 0.853 & 1.397 & -0.315 & -1.391 & 3.120 & -0.591 & -1.015 & 0.183 & -1.340 & 1.253 & 3.502 & 0.778 \\
\hline $\mathrm{Xi}$ & 2 & 1 & 1 & 2 & 1 & 1 & 2 & 1 & 2 & 3 & & & & \\
\hline$\beta X \mathrm{X}$ & -0.395 & 1.119 & 0.853 & 1.397 & -0.315 & -1.391 & 3.120 & -0.591 & -1.015 & 0.183 & -1.340 & 1.627 & 5.088 & 0.836 \\
\hline $\mathrm{Xi}$ & 1 & 0 & 0 & 0 & 0 & 1 & 0 & 0 & 2 & 3 & & & & \\
\hline$\beta X \mathrm{X}$ & -0.768 & & & & & -1.391 & & & -1.015 & 0.183 & -1.340 & -4.330 & 0.013 & 0.013 \\
\hline $\mathrm{Xi}$ & 1 & 0 & 0 & 0 & 0 & 1 & 0 & 1 & 2 & 3 & & & & \\
\hline$\beta X \mathrm{X}$ & -0.768 & & & & & -1.391 & & -0.591 & -1.015 & 0.183 & -1.340 & -4.921 & 0.007 & 0.007 \\
\hline $\mathrm{Xi}$ & 0 & 1 & 0 & 0 & 0 & 0 & 0 & 0 & 0 & 0 & & & & \\
\hline$\beta X \mathrm{X}$ & 0 & 1.119 & & & & & & & & & -1.340 & -0.220 & 0.802 & 0.445 \\
\hline $\mathrm{Xi}$ & 0 & 0 & 2 & 0 & 0 & 0 & 0 & 0 & 0 & 0 & & & & \\
\hline$\beta X \mathrm{X}$ & & & 0.885 & & & & & & & & -1.340 & -0.455 & 0.635 & 0.388 \\
\hline $\mathrm{Xi}$ & 0 & 0 & 5 & 0 & 0 & 0 & 0 & 0 & 0 & 0 & & & & \\
\hline$\beta X \mathrm{X}$ & & & 0.400 & & & & & & & & -1.340 & -0.939 & 0.391 & 0.281 \\
\hline $\mathrm{Xi}$ & 0 & 0 & 0 & 1 & 0 & 0 & 0 & 0 & 0 & 0 & & & & \\
\hline$\beta \mathrm{Xi}$ & & & & 1.397 & & & & & & & -1.340 & 0.057 & 1.059 & 0.514 \\
\hline Xi & 0 & 0 & 0 & 4 & 0 & 0 & 0 & 0 & 0 & 0 & & & & \\
\hline$\beta X \mathrm{X}$ & & & & 0.865 & & & & & & & -1.340 & -0.475 & 0.622 & 0.384 \\
\hline $\mathrm{Xi}$ & 3 & 1 & 2 & 2 & 0 & 4 & 2 & 0 & 1 & 2 & & & & \\
\hline$\beta \mathrm{Xi}$ & -0.378 & 1.119 & 0.885 & 1.397 & & -0.1860 & 3.120 & & -0.364 & 1.498 & -1.340 & 5.752 & 314.684 & 0.997 \\
\hline $\mathrm{Xi}$ & 3 & 1 & 2 & 2 & 0 & 4 & 2 & 1 & 2 & 3 & & & & \\
\hline$\beta X \mathrm{X}$ & -0.378 & 1.119 & 0.885 & 1.397 & & -0.1860 & 3.120 & -0.591 & -1.015 & 0.183 & -1.340 & 3.196 & 24.424 & 0.961 \\
\hline $\mathrm{Xi}$ & 3 & 1 & 2 & 2 & 0 & 4 & 2 & 0 & 2 & 3 & & & & \\
\hline$\beta X \mathrm{X}$ & -0.378 & 1.119 & 0.885 & 1.397 & & -0.1860 & 3.120 & & -1.015 & 0.183 & -1.340 & 3.786 & 44.101 & 0.978 \\
\hline $\mathrm{Xi}$ & 1 & 0 & 0 & 0 & 0 & 0 & 0 & 0 & 2 & 3 & & & & \\
\hline$\beta X \mathrm{X}$ & -0.768 & & & & & & & & -1.015 & 0.183 & -1.340 & -2.939 & 0.053 & 0.050 \\
\hline XI & 1 & 0 & 0 & 0 & 0 & 0 & 0 & 1 & 2 & 3 & & & & \\
\hline$\beta X \mathrm{X}$ & -0.768 & & & & & & & -0.591 & -1.015 & 0.183 & -1.340 & -3.530 & 0.029 & 0.028 \\
\hline $\mathrm{Xi}$ & 2 & 1 & 1 & 2 & 1 & 1 & 2 & 1 & 2 & 3 & & & & \\
\hline$\beta X i$ & -0.395 & 1.119 & 0.853 & 1.397 & -0.315 & -1.3911 & 3.120 & -0.591 & -1.015 & 0.183 & -1.340 & 1.627 & 5.088 & 0.836 \\
\hline $\mathrm{Xi}$ & 2 & 1 & 1 & 2 & 1 & 1 & 2 & 1 & 2 & 3 & & & & \\
\hline$\beta X \mathrm{X}$ & -0.768 & 1.119 & 0.853 & 1.397 & -0.315 & -1.3911 & 3.120 & -0.591 & -1.015 & 0.183 & -1.340 & 1.253 & 3.502 & 0.778 \\
\hline
\end{tabular}

$\beta$ :Tabla 5. Si Xi es 0; $\beta \mathrm{Xi}=0$. Fuente: construcción autores con spss 24. 
Tomando los máximos valores de las variables económicas, la probabilidad de que los estudiantes de la región Andina; educación del padre postgrado; estrato 6; ingresos familiares más de 7 millones de pesos; valor de la matrícula más de 5 millones de pesos; sin beca; cero personas a cargo; mujer; programa presencial y universidad obtenga una puntuación menor o igual a la media en el módulo es de 0.013; modificando sólo el género, hombre, la probabilidad respectiva es de 0.007, disminuye. Bajo las mismas condiciones el hombre tiene menor probabilidad de estar bajo la media en el módulo.

Analizando los $\beta_{\mathrm{i}}$ de cada variable independiente o predictora y los OR asociados (tabla 5) a cada variable, podemos concluir: que la educación del padre influye en el rendimiento del módulo de inglés, con mayor probabilidad de obtener puntuación menor o igual a la media en hijos de padres sin escolaridad además, los $\beta_{\mathrm{i}}$ son positivos para padres con escolaridad máxima, educación técnica o tecnológica sin título, pero para educación técnica o tecnológico con título o profesional con o sin título, son negativos, lo cual indica que disminuye esta probabilidad. Pero es importante analizar esto con los OR y su respectiva categoría de referencia (padres con posgrado), tomando todas las demás variables constantes, vemos que un hijo de padres sin escolaridad multiplica por 3.063 la probabilidad de los que tienen padres con posgrado, de sacar una puntuación en el módulo menor o igual a la media; pero este factor baja a 2.0074 con padres con primaria completa y a medida que aumenta la escolaridad el factor baja, la probabilidad crece pero en menor proporción. Para padres con educación técnica o tecnológica con título y profesional con o sin título, por cada año de escolaridad disminuye el OR de tener puntuación inferior a la media en un $16.36 \%$ y $14.47 \%$ respectivamente $\left[(\mathrm{OR}-1)^{*} 100\right]$. Vimos que la probabilidad de estar por debajo de la media para los $\mathrm{X}_{\mathrm{i}}=0$ fue de $20.8 \%$. Si mantenemos constante esto y tomamos padres sin escolaridad, la probabilidad aumenta y es de $44.5 \%$. No olvidar que esto es para las más altas condiciones económicas y familiares, de la región Amazónica; número máximo de personas a cargo, mujer, programas virtuales e institutos tecnológicos, con padres sin escolaridad.

Con los ingresos familiares mensuales los $\beta_{\mathrm{i}}$ son positivos y los OR son mayores que 1, la categoría de referencia es ingresos mensuales de 7 o más SMLV. Para ingresos de 1 a menos de 2 SMLV el OR es de 2.4227 veces más que el que tienen 7 o más SMLV; pero para el que está entre 5 a 7 , el factor baja a 1.4925; manteniendo todas las variables en cero. Sólo cambiando ingresos para estos dos casos las probabilidades respectivas son $38.8 \%$ y $28.1 \%$, luego a mayor ingreso menor probabilidad de tener un puntaje en inglés por debajo de la media.

$\mathrm{El}$ valor de la matrícula influye en el rendimiento, los $\beta_{\mathrm{i}}$ son positivos y OR mayores que uno, la categoría de referencia es matrícula superior a 5 millones de pesos; para las matrículas entre 500 mil y un millón de pesos, la probabilidad de estar por debajo de la media en la prueba crece en una razón de 4.0429 del valor de referencia, para las matrículas de 3 a 5 millones de pesos el factor baja, es de 2.3750; mirando las probabilidades para las categorías de referencia, sólo modificando la matrícula se observan probabilidades de $51.4 \%$ y $38.4 \%$ respectivamente de tener rendimiento inferior a la media; luego a mayor matrícula tienen menor probabilidad. Para el tipo de institución encontramos que las instituciones técnicas profesionales presentan la mayor probabilidad de tener menor rendimiento, probabilidad que disminuye si es institución universitaria o universidad. El estrato presenta OR superiores a 1, la categoría de referencia es estrato 6; vemos que en el estrato 1 tienen 22.6544 veces más de posibilidad de ubicar su rendimiento por debajo de la media que el del estrato 6; el estrato 2 un 14.9128, el del estrato 5 es un 5.0742 veces más; así, a medida que aumenta el estrato la probabilidad de estar por debajo de la media disminuye. 
Las otras variables tienen un influencia inversa, a mayor valor menor probabilidad; dentro de ellas está la región geográfica donde se ubica el programa; las que presentan la mayor probabilidad de estar por debajo de la media son: Amazonía, Pacífica, Caribe, Orinoquía y Andina; para las regiones la probabilidad decrece respectivamente con respecto a la Amazonía que es la categoría de referencia en $31.46 \% ; 32.60 \%$ y $53.6 \%$, los de la región Andina tienen mayor probabilidad de ubicarse por encima de la media. El número de personas a cargo del estudiante tiene también una influencia negativa y como sus OR son menor a uno, vemos que la probabilidad decrece en menor proporción con respecto a los que tienen 5 o más personas a cargo; esto es, para los que no tienen personas a cargo decrece un $75.12 \%$ y para los que tienen $1,2,3$ y 4 personas decrece en $59.25 \%, 49.29 \%, 16.98 \%$ y $29.6 \%$ respectivamente; a mayor número de personas a cargo la probabilidad decrece en menor proporción. El tener beca y el género (hombre) influye negativamente; si es hombre la probabilidad decrece en un $44.62 \%$ con respecto a la mujer y si tienen beca decrece en un $27 \%$ con respecto al que no la tiene.

En la tabla 6 se muestra la probabilidad que el estudiante tenga un rendimiento en la prueba menor o igual a la media si el estudiante es de la región Pacífica; padre sin educación; ingresos familiares mensuales entre 1 a 2 millones de pesos mensuales; valor de la matrícula entre 500 mil y un millón de pesos, no tienen beca; tiene tres personas a cargo; del estrato 1 ; mujer, método del programa a distancia e institución técnica profesional es de $99.7 \%$. Esta probabilidad es para los máximos valores de los OR que hacen crecer multiplicativamente la probabilidad. Modificando el género (hombre), el método del programa presencial y universidad la probabilidad baja a $96.1 \%$. Si el método del programa y el tipo de institución sigue igual pero el estudiante es mujer la probabilidad de estar por debajo de la media es de $97.8 \%$, parece que las mujeres de región Pacífica tienen menor rendimiento en el examen.
Tomando las categorías de referencia donde los $\mathrm{X}_{\mathrm{i}}=0$, la probabilidad de tener una puntuación menor o igual a la media es de $20.8 \%$. Luego la probabilidad de tener una probabilidad superior a la media para estos estudiantes es de $79.2 \%$. Si modificamos sólo la región por la Andina; método presencial y de universidad, la probabilidad cae a $5 \%$. Luego, la probabilidad de ubicarse por encima de la media para este grupo es del 95\%. Pero si mantenemos constantes todas las condiciones anteriores y modificamos el género por hombre, la probabilidad baja 2.8\%, esto es el $97.2 \%$ de ubicarse por encima de la media, se espera que los hombres tengan mejor rendimiento en la prueba en estas condiciones.

Ahora, tomando las menores condiciones socioeconómicas y familiares, pero con programas presenciales y universidades, se calculó la probabilidad de obtener puntuación menor o igual a la media para estudiantes de la región Caribe; padre sin educación; ingresos familiares menores a $1 \mathrm{SMLV}$; matrícula entre 500 mil y un millón de pesos, con beca; cero personas a cargo; estrato 1; hombre; programa presencial y de una universidad, tienen un $83.6 \%$ de probabilidad, pero si es de la región Andina es del $77.8 \%$.

\section{Conclusiones}

En esta investigación, el objetivo es determinar las variables familiares, económicas, personales, institucionales y del programa, que pueden influir y que permitan clasificar correctamente los resultados de la prueba SaberPro del módulo de inglés, que presenta todo aspirante para optar por título en la educación superior. En nuestro caso, la variable dependiente dicotómica utilizada en la regresión logística que se aplicó (ver fórmula 5), identificó como éxito tener una puntuación menor o igual a la medía $\mathrm{Y}=1, \mathrm{y}$ tener una puntuación mayor a la media se codificó con $\mathrm{Y}=0$.

Este modelo presenta una sensibilidad alta del $89.1 \%$, por lo que clasifica como muy bien a los 
estudiantes que están por debajo de la media, y sólo un $49.3 \%$ para los que están por encima de la media, el modelo tiene una exactitud del $75 \%$ en los datos seleccionados, y en la muestra de validación un $73 \%$. La probabilidad de que en el modelo entre un estudiante con puntuación en el examen menor o igual a la media, y otro con puntuación superior a la media, sea clasificado correctamente mediante una estimación puntual es del 78\%, además, la capacidad de clasificación del modelo mediante un intervalo de confianza al $95 \%$ está entre $76 \%$ y $79.5 \%$, que se asume como buena, con un umbral del $67.09 \%$. La probabilidad para todos $\operatorname{los} \mathrm{X}_{\mathrm{i}}=0$ (que corresponde a la categoría de referencia de cada variable en el modelo) de ubicarse por debajo de la media es de 20.8\%; esto es para estudiantes de la Amazonía; educación del padre posgrado, ingresos familiares de 7 o más SMV, matrículas del año anterior superiores a 5 millones de pesos, estudiante sin beca, con 5 o más personas a cargo, del estrato 6, mujer, método del programa virtual e institución tecnológica.

Teniendo en cuenta las variables seleccionadas de la tabla 5 y de acuerdo con el signo de los $\beta$ i, el valor de los OR y la variable categórica de referencia, se puede afirmar que hay variables que incrementan la probabilidad de tener una puntuación en el módulo de inglés inferior o igual a la media, si los $\beta$ i son positivos y sus OR son mayores que uno, o disminuyen si éstos son negativos o menores a uno.

Las conclusiones por variable en el modelo se presentan a continuación:

Educación del padre: existen dos comportamientos en esta variable: 1) para los casos de padres con educación que va desde sin escolaridad hasta secundaria completa o técnica o tecnológica sin título, hay una relación directa. De este bloque, el grupo que no tiene escolaridad muestra mayor valor, seguido por los que terminaron secundaria o tienen estudios técnicos o tecnológicos sin título. De acuerdo con la categoría de referencia de la variable, que es el grupo de padres con posgrado, se ve que los hijos de padres con menor educación tienen mayor probabilidad de quedar por debajo de la media que los hijos de padres con mayor educación. Esto nos lleva a afirmar que los padres con menor educación posiblemente no muestran a sus hijos la necesidad de aprender una segunda lengua. 2) Para hijos de padres con educación técnica o tecnológica con título o educación profesional con o sin título, la probabilidad decrece con respecto a los que tienen padres con posgrado. Estos padres que tienen nivel de educación más alto pueden mostrar a sus hijos la necesidad de la segunda lengua. Luego la educación del padre con un nivel máximo técnico o tecnológico sin título implica que en el módulo quedan por debajo de la media, pero para padres con nivel técnico o tecnológico con título o estudios de pregrado con o sin título sí mejora el rendimiento del hijo en las pruebas de inglés. Lo cual valida lo expresado por Nikolov (2009) y Piso (2013), los antecedentes académicos del padre se correlacionan con los resultados de sus hijos.

Ingresos familiares: afectan la probabilidad positivamente, la categoría de referencia es ingresos de 7 o más SMLV, los ingresos de 1 a 2 salarios presentan la mayor probabilidad que en el módulo se ubique por debajo de la media. Pero comparando cada nivel de ingresos con respecto a la categoría de referencia, se puede concluir que la posibilidad de ubicarse por debajo de la media disminuye a medida que aumenta el ingreso.

Valor anual de la matrícula del año anterior: también influye positivamente la ocurrencia de un resultado por debajo de la media, la característica de referencia es matrícula del año anterior de 5 o más millones de pesos anuales. La matrícula que tiene más posibilidad de ubicarse por debajo de la media en el resultado del módulo de inglés, es matrícula anual entre 500 mil a 1 millón de pesos. Al comparar las matrículas con respecto a la de referencia, se puede concluir que el costo de la matrícula no asegura ubicarse en la prueba por encima de la media, pero entre más costosa sea, ayuda a disminuir la probabilidad. 
Estrato: afecta positivamente el rendimiento en el módulo. El estrato 2 es el que tiene mayor probabilidad de quedar en la prueba por debajo de la media. La categoría de referencia es estrato 6; la probabilidad de ubicarse por debajo de la media va decreciendo a medida que incrementa el estrato.

Carácter académico de la institución: la probabilidad de quedar en el módulo por debajo de la media es positiva. La característica de referencia es institución tecnológica. La institución que mayor probabilidad tiene de ubicarse por debajo de la media es la técnica-profesional, pero si es institución universitaria o universidad, la probabilidad de ubicarse por debajo de la media disminuye con respecto a las instituciones tecnológicas.

Las variables económicas (ingresos familiares, valor anual de la matrícula y estrato) influyen en el resultado de la prueba, la probabilidad de ubicarse por debajo de la media crece con respecto a la probabilidad de las variables categorías de referencia, pero a medida que mejora la situación económica esta probabilidad decrece. Las condiciones económicas ayudan pero no aseguran un buen resultado. La educación del padre influye, sobre todo los que son técnicos o tecnólogos o con estudios de pregrado con o sin título, este grupo ya por su nivel de educación podría pensarse que son conscientes de la necesidad del segundo idioma y que sus hijos lo perciben así. Así, factores externos como cultura y estatus, según Della Chiesa et al. (2012), afectan el aprendizaje.

Las siguientes variables tienen una relación inversa con la puntuación en la prueba.

Fuente de los recursos con los que se canceló la matrícula del año anterior - beca o subsidio: baja la probabilidad de ubicarse por debajo de la media. La categoría de referencia es no tenerla. Parece que el estudiante con beca o subsidio tiene una presión o motivación de presentar un buen resultado, se espera que mejore el rendimiento del estudiante respecto al que no la tiene.

Género: disminuye la probabilidad de ubicarse por debajo de la media. La categoría de referencia es mujer. Los datos muestran que los hombres pueden presentar un mejor rendimiento en la prueba que las mujeres.

Método del programa: disminuye la probabilidad a medida que el contacto del estudiante con el docente es más cercano. La categoría de referencia es virtual. La menor probabilidad se presenta para el método presencial.

Número de personas a cargo del estudiante: la categoría de referencia es 5 personas o más. La categoría que presenta menor probabilidad con respecto a la categoría de referencia es cero personas. A medida que aumenta el número de personas a su cargo, aumenta la probabilidad de obtener peores resultados en la prueba.

Región de ubicación del programa: la categoría de referencia es la región Amazónica. La probabilidad de obtener mejores resultados es mayor en la región Andina. En su orden van decreciendo las probabilidades para Orinoquía, Caribe y Pacífico.

Se puede concluir que el no tener personas a cargo, es decir, sólo responder por sí mismo, estudiar en programas con metodologías presenciales, ubicarse en regiones de mayor desarrollo, ser hombre, estudiar con beca o subsidio y con padres con educación técnica o tecnológica con título o educación profesional con o sin título, disminuye la probabilidad de ubicarse por debajo de la media. Si además de esto, se ayuda con mejores condiciones económicas, como de estrato 5, estudiando en universidad, ingresos mensuales entre 5 a 7 millones de pesos, matrículas entre 3 a 5 millones de pesos, los resultados del SaberPro en el módulo de inglés mejoran para el estudiante.

Dando una mirada a la importancia que tiene actualmente para los programas de administración y afines el aprendizaje de una segunda lengua, este trabajo nos muestra que el Estado y las instituciones educativas deben revisar la metodología del programa. La presencialidad disminuye la probabilidad de quedar por debajo de la media, lo cual nos llevaría a pensar que el docente juega un papel importante en 
el rendimiento del estudiante. Las bajas condiciones económicas de los estudiantes influyen en el rendimiento, luego se recomienda que para estos grupos se brinden facilidades económicas que suplan las fortalezas que sí tienen los estudiantes de estratos altos. También muestra que entre más personas tengan a cargo, es más probable quedar por debajo de la media, esto es, tienen menor capacidad económica para invertir en su segunda lengua por sus responsabilidades. Las regiones afectan los resultados y se resalta que la región Andina es la que tiene menos estudiantes por debajo de la media; esta región es posiblemente la que más inversión y soporte del Estado

\section{Referencias}

Bacha, N. y R. Bahous (2008), "Contrasting views of business students' writing needs in an EFL Environment", English for Specific Purposes, vol. 27, núm. 1, pp. 74-93.

Berlanga-Silvente, V. y R. Vilà-Baños (2014), "Cómo obtener un modelo de regresión logística binaria con SPSS", REIRE, Revista d'Innovació i Recerca en Educació, vol. 7, núm. 2, pp. 105-118.

Bhatia, V. K. y S. Bremner (2012), "English for business communication", Language Teaching, vol. 45, núm. 4, pp. 410-45.

Cárdenas, R. y N. Miranda (2014), "Implementación del Programa Nacional de Bilingüismo en Colombia: un balance intermedio", Educación y Educadores, vol. 17, núm. 1, pp. 51-67.

Charles, M. (2007), "Language matters in global communication: article based on ORA lecture, october 2006”, Journal of Business Communication, vol. 44, núm. 3, pp. 260-82.

Chiswick, B. (2008), "The economics of language: an introduction and overview", IZA Discussion Paper, núm. 2568 . puede tener. Como algo interesante, el género también influye en el rendimiento, pues parece que los hombres tienen mejor rendimiento con respecto a las mujeres. Este trabajo recomienda que las instituciones educativas y estudiantes, den una mirada a los factores involucrados en este estudio. Se partió de 26 variables y de acuerdo con un proceso iterativo sólo quedaron 10. No se puede olvidar que dada la información suministrada por la prueba SaberPro, no se cuenta con información intrínseca, como puede ser la motivación del estudiante, que es un factor predominante en el aprendizaje de una lengua, según citán diferentes autores.

Della Chiesa, B.,J. Scott y C. Hinton (eds.) (2012), Languages in a global world: learning for better cultural understanding, París, OECD Publishing.

Fandiño Parra, Y.,J. Bermúdez-Jiménez y V. Lugo Vásquez (2012), "Retos del Programa Nacional de Bilingüismo", Educación y Educadores, vol. 15, núm. 3, pp. 363-81.

Fuertes-Olivera, P.A. y S. Gómez-Martínez (2004), "Empirical assessment of some learning factors affecting Spanish students of business english", English for Specific Purposes, vol. 23, núm. 2, pp. 163-80.

García-Esteban, S. (2013), "Análisis y evaluación de cursos de inglés de los negocios a distancia asistidos por las nuevas tecnologías de la información y la comunicación", tesis doctoral, España, Facultad de Filosofia y Letras, Universidad de Alcalá.

Gardner, R. (1983), "Learning another language: a true social psychological experiment", fournal of Language and Social Psychology, vol. 2, núms. 2-3-4, pp. 219-239.

Gardner, R. (1985), The social psychology and second language learning role of attitudes and motivation, Londres, Edward Arnold. 
Kankaanranta, A. y L. Louhiala-Salimen (2013), "What language does global business speak?- The concept and development of BELF", Iberica, vol. 26, pp. 17-34.

Knapp, K. y G. Meierkord (eds.) (2002), Lingua Franca Communication, Frankfurt am Main, Peter Lang.

Kormos, J. y T. Kiddle (2013), "The role of socioeconomic factors in motivation to learn English asa foreign language: the case of Chile", System, vol. 41, núm. 2, pp. 399-412.

Lamb, M. (2012), "A self system perspective on young adolescents' motivation to learn English in urban and rural settings", Language Learning, vol. 62, núm. 4, pp. 997-1023.

Lightbown, P. M. y N. Spada (2006), How languages are learned (3rd ed.), Oxford, Oxford University Press.

McManus, W., W. Gould y F. Welch (1983), "Earnings of Hispanic men: the role of English language proficiency", Fournal of Labor Economics, vol. 1, núm. 2, pp. 101-130.

Mejía-Mejía, S. (2016), “¿Vamos hacia una Colombia bilingüe? Análisis de la brecha académica entre el sector público y privado en la educación del inglés", Educación y Educadores, vol. 19, núm. 2, pp. 223-37.

Ministerio de Educación Nacional (MEN) (2014), Colombia Bilingüe, <http://aprende.colombiaaprende.edu.co/ colombiabilingue $>$ [Consulta: junio de 2017].

Mitchell, R. y F. Myles (2004), Second language learning theories, 2nd edition, Londres, Hodder Arnold.

Moody, R. (1988), "Personality preferences and foreign language learning", Modern Language fournal, vol. 72, núm. 4, pp. 389-401.

Morrison, D. (1972), "Upper bounds for correlations between binary outcomes and probabilistic", fournal of the American Statistical Association, vol. 67, núm. 337, pp. 68-70.

Nickerson, C. (2005), "English as a lingua franca in international business contexts", English for Specific Purposes, vol. 24, núm. 4, pp. 367-80.

Nikolov, M. (2009), "Early modern foreign language programmes and outcomes: factors contributing to Hungarian learners' proficiency", en M. Nikolov (ed.), Early learning of modern foreign languages. Multilingual matters, Bristol, pp. 90-107.
Nteliou, E. y O. Kehagia (2016), "Intercultural perceptions among undergraduate students in English for Business and Economics courses", International fournal of Language, Translation and Intercultural Communication, vol. 4, pp. 98-109.

Programme for International Student Assessment (PISA) (2003), Learning for tomorrow's world. First results from PISA 2003, París, OECD Publishing.

República de Colombia (2014), Plan Nacional de Desarrollo 2014-2018, Todos Por Un Nuevo País, <https:// colaboracion.dnp.gov.co/CDT/PND/PND20142018Tomolinternet.pdf> [Consulta: junio de 2017].

Reyes, J., C. Escobar, J. Duarte y P. Ramírez (2007), "Una aplicación del modelo de regresión logística en la predicción del rendimiento estudiantil", Estudios Pedagógicos, vol. XXXIII, núm. 2, pp. 101-120.

Richards, J. y T. Rodgers (2001), Approaches and methods in language teaching, (2nd ed.) Cambridge, Cambridge University Press.

Rodríguez-Chamussy, L., L. Lopez-Calva y K. Miyamoto (2012), "Economic incentives for language acquisition", en Bruno Della Chiesa, Jessica Scott and Christina Hinton, (eds.), Languages in a global world: learning for better cultural understanding, París, OECD Publishing.

Rogerson-Revell, P. (2007), "Using English for international business: a European case study", English for Specific Purposes, vol. 26, núm. 1, pp. 103-20.

Rueda-Cataño, M. C. y M. Wilburn (2014), "Enfoques teóricos para la adquisición de una segunda lengua desde el horizonte de la práctica educativa", Perfiles Educativos, vol. XXXVI, núm. 143, pp. 21-28.

Salas, M. (1996), "La regresión logística. Una aplicación a la demanda de estudios universitarios", Estadística Española, vol. 38, núm. 141, pp. 193-217.

Sánchez-Jabba, A. (2013), "Bilingüismo en Colombia", Documentos de Trabajo Sobre Economía Regional, Cartagena, Banco de La República, Centro de Estudios Económicos Regionales (CEER), núm. 191, p. 46, <http://banrepc.dayscript.com/sites/default/files/ publicaciones/archivos/dtser_191.pdf $>$ [Consulta: junio de 2017]. 
Santana, C., A. García-Santillán y M. Escalera-Chávez (2016), "Variables que influyen sobre el aprendizaje del inglés como segunda lengua", Revista Internacional de Lenguas Extranjeras, núm. 5, pp. 79-94.

St. John, M. (1996), "Business is booming: business English in the 1990s", English for Specific Purposes, vol. 15 num. 1, pp. 3-18.

Tainer, E. (1988), "English language proficiency and the determination of earnings among foreign-born men", The Fournal of Human Resources, vol. 23, núm. 1, pp. 108122.

Trinder, R. (2013), "Business students' beliefs about language learning in a university context", English for Specific Purposes, vol. 32, núm. 1, pp. 1-11.

Trinder, R. y M. Herles (2011), 'Students' and teachers' notions of effective (business) English teaching: global and local influences", en Robert McColl Millar y Mercedes Durham (eds.), Applied linguistics, global and local. Proceedings of the 43 annual meeting of the British Association for Applied Linguistics, pp. 351-361.
Valenzuela, M. J., K. Romero, C. Vidal-Silva y A. Philominraj (2016), "Factores que influyen en el aprendizaje del idioma inglés de nivel inicial en una universidad chilena", Formación Universitaria, vol. 9, núm. 6, pp. 63-72.

Waner, K. (1995), "Competencies needed by employees as perceived by business faculty and business professionals", Business Communication Quarterly, vol. 58, núm. 4, pp. 5157.

Zagan-Zelter, D. y S. Zagan-Zelter (2010), "Teaching business English - A challenge both for students and academics", Procedia - Social and Behavioral Sciences, vol. 3, núm. 1, pp. 245-50.

Zhang, Z. (2013), "Business English students learning to write for international business: what do international business practitioners have to say about their texts?", English for Specific Purposes, vol. 32, núm. 3, pp. 144-56.

Zhou, G. y G. Griffiths (2011), "Intercultural communicative competence", English Language and Literature Studies, vol. 1 núm. 2, pp. 113-122.

\section{Cómo citar este artículo:}

Cabeza, Leonor, Jahir Lombana y Jaime Castrillón (2020), "Factores externos en el desempeño de pruebas genéricas de Estado (SaberPro) de inglés en estudiantes de administración y afines en Colombia", Revista Iberoamericana de Educación Superior (RIES), vol. XI, núm. 30, pp. 182-200, https://doi.org/10.22201/iisue.20072872e.2020.30.595 [Consulta: fecha de última consulta]. 